\title{
Performance of broadband tristable energy harvesters
}

\author{
Shengxi Zhou ${ }^{1}$, Grzegorz $\operatorname{Litak}^{2,3, *}$, and Junyi $\mathrm{Cao}^{4}$ \\ ${ }^{1}$ School of Aeronautics, Northwestern Polytechnical University, Xi' an, 710072, China \\ ${ }^{2}$ Lublin University of Technology, Faculty of Mechanical Engineering, Nadbystrzycka 36, PL-20-618 \\ Lublin, Poland \\ ${ }^{3}$ Department of Process Control, AGH University Science and Technology, Department of Process \\ Control, PL Mickiewicza 30, PL-30-059 Krakow, Poland \\ ${ }^{4}$ School of Mechanical Engineering, Xi'an Jiaotong University, Xi'an, 710049, China
}

\begin{abstract}
We analyze dynamical responses of energy harvesters with a mechanical resonator possessing three potential wells. The frequency spectral analysis of simulated systems demonstrates that additional sub-harmonic and super-harmonic resonances appear. These additional solutions make the frequency broadband effect and effectively increase the voltage output. We show the characteristic features of the obtained solutions. Appearance of coexisting periodic solutions and chaotic solutions are also shortly discussed.
\end{abstract}

\section{Introduction}

Currently, vibration energy harvesting from low-level ambient vibrations has been received more and more attention [1-5]. The characteristics and frequency broadband performance of nonlinear monostable and bistable energy harvesters have been numerically and experimentally revealed and verif ed [6-12]. Due to distinct advantages over monostable and bistable oscillators, a new class of broadband energy harvesters with three potential wells [13-15] has been proposed.

\section{Modelling and numerical analysis}

Figure 1 shows the schematic diagram of the tristable energy harvester (TEH) in this paper. It is shown that the harvester mainly includes a piezoelectric beam with a tip magnet, two external magnets, a load resistance and a supporting mechanism. The nonlinear magnetic force can be produced by the interaction among the tip magnet and two external magnets, which depends on the relative position of these magnets. Therefore, the equivalent nonlinear restoring force of the TEH is the vector sum of the linear restoring force of the piezoelectric beam and the nonlinear magnetic force. It can be found that the TEH has as many as fve equilibrium positions (1, 3 and 5 are stable equilibrium positions; 2 and 4 are unstable equilibrium positions). Under the base acceleration excitation, the nonlinear electromechanical model of the THE can be described by the following equations:

\footnotetext{
*e-mail: g.litak@pollub.pl
} 


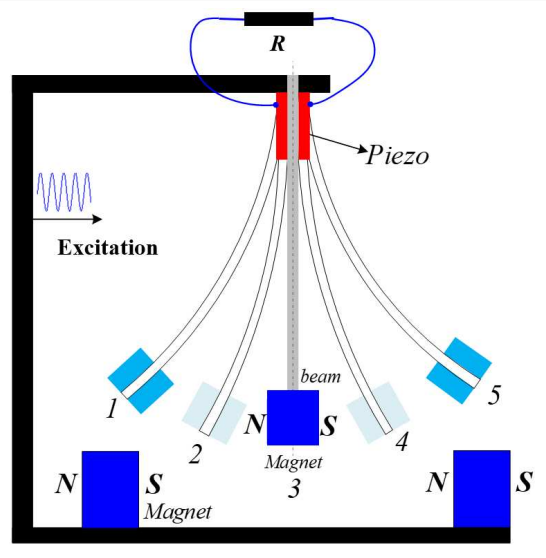

Figure 1. Schematic picture of the energy harvesting system.

$$
\begin{aligned}
& \ddot{x}(t)+\xi \dot{x}(t)-F_{r}(x)-\theta v(t)=A \cos (\omega t), \\
& C_{p} \dot{v}(t)+v(t) / R+\theta \dot{x}(t)=0,
\end{aligned}
$$

where $\xi$ is a dimensionless damping coefficient. $F_{r}(x)$ is a restore force, which can be expressed as:

$$
F_{r}(x)=-x+\mu x^{3}-\beta x^{5} .
$$

$\mu$ and $\beta$ are coefficients of the cubic $\left(x^{3}\right)$ and quintic terms $\left(x^{5}\right)$ of the equivalent nonlinear restoring force, respectively. The linear term coefficient standing by $-x$ is set to be one for analysis. In order to make f ve roots of the function $F_{r}(x)$ (to yield a tristable characteristic), $\mu$ and $\beta$ should meet the specif c conditions which can be deduced based on Eq. (5). Then, the potential energy function can be expressed as:

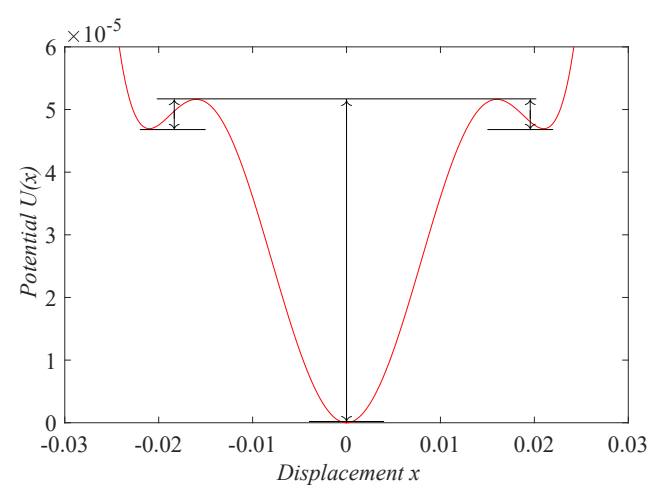

Figure 2. Tristable system potential wells. The arrows indicate the potential barriers to escape from the particular potential wells.

$$
U(x)=x^{2} / 2-\mu x^{4} / 4+\beta x^{6} / 6
$$


(a)

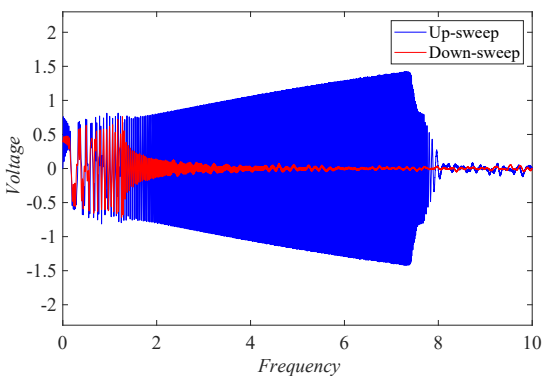

(b)

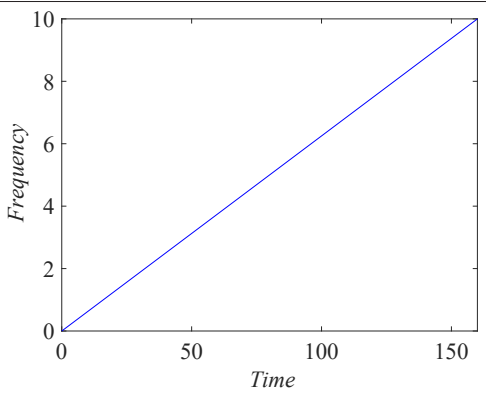

Figure 3. (a) Voltage output through the frequency sweeps for nodal initial conditions at the lower and higher frequencies $[x, \dot{x}, v]=[0,0,0]$, (b) time versus frequency in sweep up. Frequency, time, and voltage are in dimensionless units.

(a)

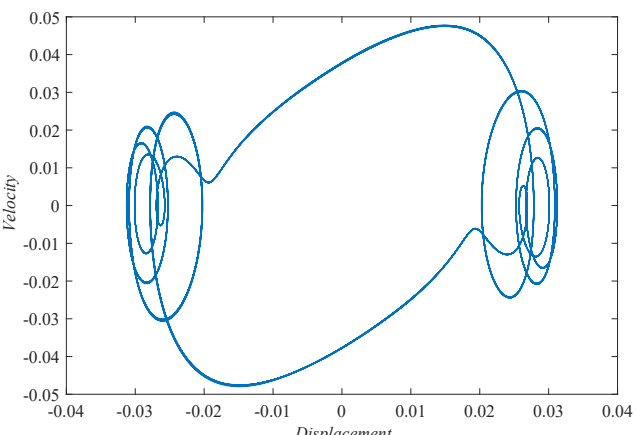

(b)

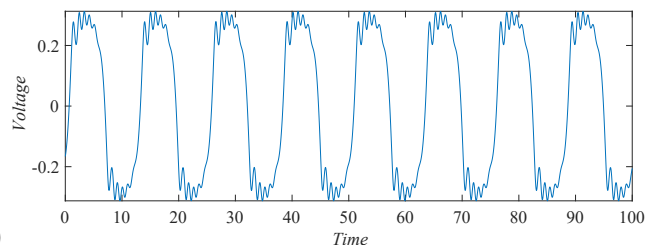

(c)

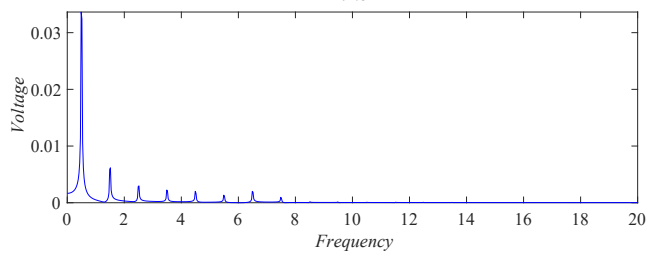

Figure 4. (a) 2D phase portrait, (b) time history of voltage output, (c) Voltage output spectrum for excitation frequency $\omega=0.5$ and initial conditions $[x, \dot{x}, v]=[0,0,0]$. Frequency, time, displacement, velocity and voltage, are in dimensionless units.

It is noted that all the parameters are dimensionless for numerical analysis, while these parameters can be theoretically calculated or experimentally measured based on a specif c energy harvesting device. In this study, the dimensionless system parameters are set to: $\xi=$ $0.8, C_{p}=0.05, \theta=0.5, R=1 \times 10^{7}, \mu=6173.8, \beta=8857709.8$. As shown in Figure 2, different from nonlinear monostable and bistable energy harvesters, the TEH has as many as three potential wells, and two of them are symmetrical and have the same depth in this paper. 
(a)
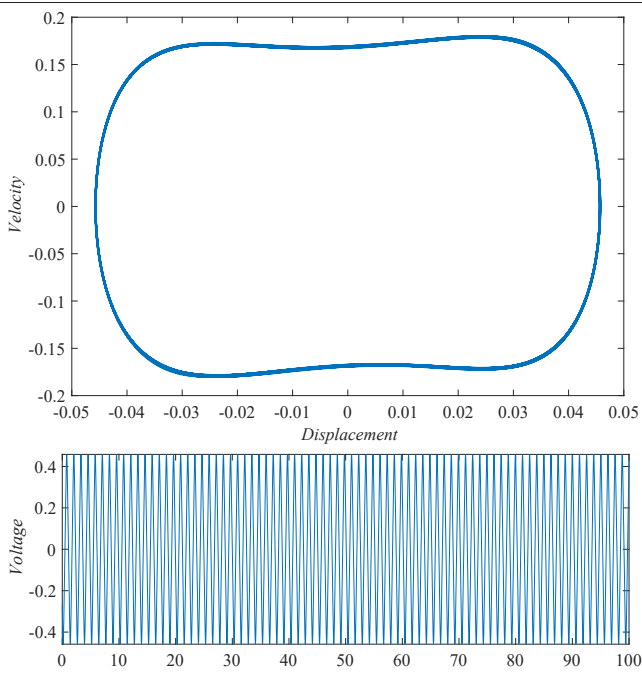

(b)

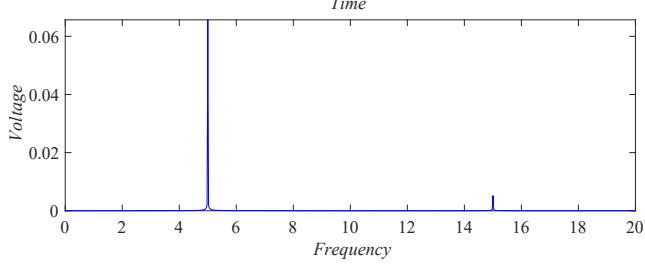

Figure 5. (a) 2D phase portrait, (b) time history of voltage output, (c) Voltage output spectrum for excitation frequency $\omega=5$ and initial conditions $[x, \dot{x}, v]=[0,0,0]$. Frequency, time, displacement, velocity and voltage, are in dimensionless units.

When the excitation level is low, the oscillations of the TEH will be limited to one potential well following low-energy intrawell oscillations. When the excitation level is high enough, the TEH will overcome their potential barriers and realize high-energy interwell oscillations (which means that oscillation amplitude is large, and the motions cross all the potential wells). Therefore, in order to investigate nonlinear characteristics of the TEH, the numerical analysis should be provided.

The equations of motion, Eqs. (1)-(2), are used in simulations of the system. At $\mathrm{frst}$, we perform calculations of non-stationary frequency sweep conditions. Voltage output (Fig. 3a) is ploted against frequency which is increased (Fig. 3b) or decreased with time. One can see different voltage paths for increasing and decreasing frequencies which are typical for nonlinear systems. For better clarity, we perform studies of stationary cases (Figs. 4-7), plotting the phase portrait projected to the corresponding displacement-velocity plane and voltage response in time and frequency domains.

In Fig. 4, we show the results for low frequency $\omega=0.5$ with nodal initial conditions. Although, the low frequency dominates in the response (Figs. 4b-c) the small super-harmonic frequencies of multiple orders, with respect to the excitation frequencies, are also present. Finally, in the phase portrait, one can see a number of small loops around local minimum of the potential and then jump to the other potential well. In the time series, this effect is related to small corrugation imposed on the leading periodic response with the excitation period. One should note that this is a nonlinear combination of large and small orbits. 
(a)
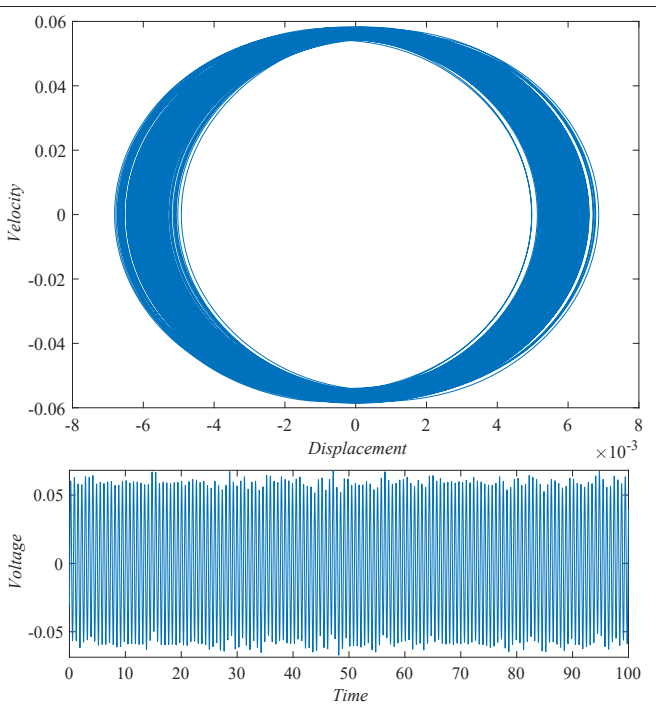

(b)

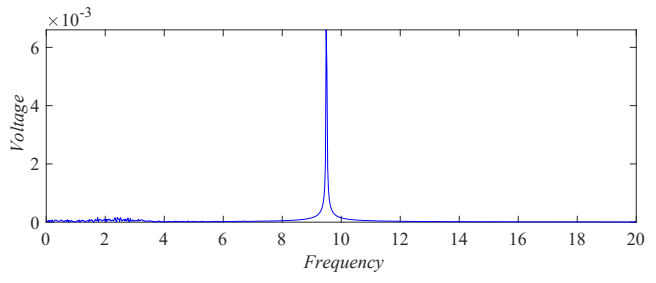

Figure 6. (a) 2D phase portrait, (b) time history of voltage output, (c) Voltage output spectrum for excitation frequency $\omega=9.5$ and initial conditions $[x, \dot{x}, v]=[0,0,0]$. Frequency, time, displacement, velocity and voltage, are in dimensionless units.

By increasing the excitation frequency, we obtain a single orbit response ( $\omega=5$ in Fig. 5). Note that the shape of the orbit is not circular (Fig. 5a) which is also a typical feature for nonlinear systems. Consequently, the Fourier spectrum has a small super-harmonic component of $2 \omega$. In Fig. 6 we plot the corresponding results for $\omega=9.5$ and the nodal initial conditions. Interestingly, this response is not periodic. It is presumably chaotic with the excitation frequency as a leading frequency in the response and chaotic modulation with smeared frequencies ranges $\omega \in[1.5,3]$ (see Fig. 6c). However, to tell more about the nature of this solution, additional tests should be performed. The other interesting feature of this solution is that the size of the orbit on the phase portrait is fairly small (Fig. 6a). Therefore this solution is entirely contained in the middle potential well (see Fig. 2). Consequently, the voltage output is small as well. This solution is not unique in the assumed set of system parameters. Another solution can be easily found for different initial conditions. In Fig. 7, we show such a solution for $[x, \dot{x}, v]=[0.06,0,0]$. This solution represents a large orbit as possible to see in Fig. 7a. The leading response frequency is two times smaller, therefore, the response can be called as a sub-harmonic $1 / 2$ resonance solution.

\section{Conclusions}

We analyzed dynamical responses of energy harvesters with a mechanical resonator possessing three potential wells. The main advantage of this system is the appearance of additional 
(a)
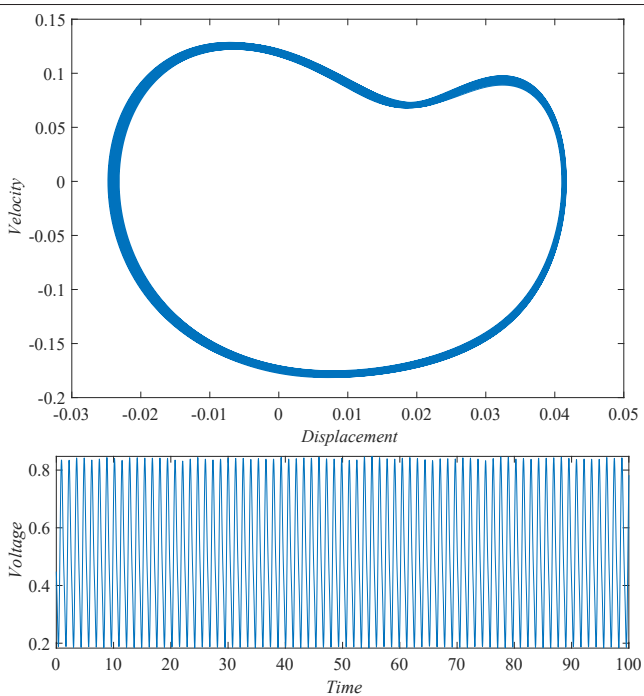

(b)

(c)

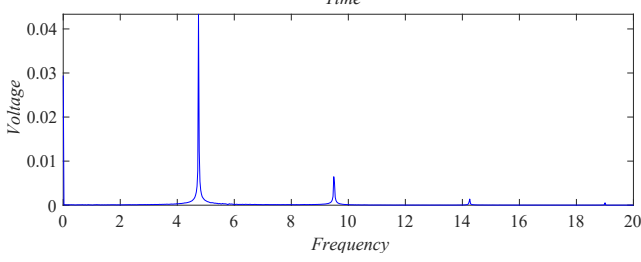

Figure 7. (a) 2D phase portrait, (b) time history of voltage output, (c) Voltage output spectrum for excitation frequency $\omega=9.5$ and initial conditions $[x, \dot{x}, v]=[0.06,0,0]$. Frequency, time, displacement, velocity and voltage, are in dimensionless units.

solutions absent in the linear system. These are sub-harmonic, super-harmonic and chaotic solutions. In contrast to the bistable system where dominates odd sub-harmonic solutions [8], the considered TEH show the even (namely period 2) sub-harmonic solution with a large orbit where the symmetry of the attractor is broken by the nonlinear effects of TEH (see Fig. 7).

\section{References}

[1] S.P. Beeby, M.J. Tudor, N.M. White, Meas. Sci. Technol. 17, R175 (2006)

[2] P.D. Mitcheson, E.M. Yeatman, G.K. Rao, A.S. Holmes, T.C. Green, Proceedings of the IEEE 96, 1457 (2008)

[3] R.L. Harne, K.W. Wang, Smart Mat. Struct. 22, 023001 (2013)

[4] S.P. Pellegrini, N. Tolou, M. Schenk, J.L. Herder, J. Intell. Mater. Syst. Struct. 24, 1303 (2013)

[5] J. Twiefel, H. Westermann, J. Intell. Mater. Syst. Struct. 24, 1291 (2013)

[6] M.F. Daqaq, R. Masana, A. Erturk, D.D. Quinn, Applied Mechanics Reviews 66, 040801 (2014)

[7] A. Syta, G. Litak, M.I. Friswell, S. Adhikari, Eur. Phys. J. B 89, 99 (2016)

[8] T. Huguet, A. Badel, A., M. Lallart, Appl. Phys. Lett. 111, 173905 (2017)

[9] M.I. Friswell, S.F. Ali, S. Adhikari, A.W. Lees, O. Bilgen, G. Litak, J. Intell. Mater. Syst.

Struct. 23, 1505 (2012) 
[10] A. Erturk, J. Hoffmann, D.J. Inman, Appl. Phys. Lett. 94, 254102 (2009)

[11] F. Cottone, H. Vocca, L. Gammaitoni, Phys. Rev. Lett. 102, 080601 (2009)

[12] G Litak, MI Friswell, S Adhikari Appl. Phys. Lett. 96, 214103 (2010)

[13] S. Zhou, J. Cao, D.J. Inman, J. Lin, S. Liu, Z. Wang. Appl. Energy 133, 33 (2014)

[14] S. Zhou, L. Zuo, Commun. Nonlinear. Sci. 61, 271 (2018)

[15] S. Zhou, J. Cao, G. Litak, J. Lin. tm-Tech. Mess. https:// doi.org/10.1515/teme -20170076 (2018) 